\title{
DCE-MRI-derived measures of tumor hypoxia and interstitial fluid pressure predict outcome in cervical carcinoma
}

\author{
Trude G. Simonsen, PhD, ${ }^{*}$ Kjersti V. Lund, MD, ${ }^{*, \dagger}$ Tord Hompland, PhD, ${ }^{*}$ \\ Gunnar B. Kristensen, PhD, ${ }^{\ddagger, \S, \|}$ and Einar K. Rofstad, PhD*
}

\footnotetext{
*Group of Radiation Biology and Tumor Physiology, Department of Radiation Biology, Institute for Cancer Research;

${ }^{\dagger}$ Department of Radiology and Nuclear Medicine; ${ }^{\ddagger}$ Department of Gynecological Cancer; and ${ }^{\S}$ Institute for Cancer Genetics and Informatics, Oslo University Hospital, Oslo, Norway; and "Institute for Clinical Medicine,

University of Oslo, Oslo, Norway
}

Short Title: DCE-MRI of tumor hypoxia and IFP.

Corresponding Author: Einar K. Rofstad, Department of Radiation Biology, Institute for Cancer Research, Norwegian Radium Hospital, Box 4953 Nydalen, 0424 Oslo, Norway.

E-mail: einar.k.rofstad@rr-research.no. Phone: +472278 1206.

Conflicts of Interest: None.

Funding: This work was supported by the Norwegian Cancer Society and the South-Eastern Norway Regional Health Authority. The sponsors were not involved in the study design; in the collection, analysis, and interpretation of data; in the writing of the report; and in the decision to submit the article for publication. 


\section{Abstract}

Purpose: Poor outcome of locally advanced cervical cancer is associated with extensive hypoxia and high interstitial fluid pressure (IFP) in the primary tumor. In this study, measures of tumor hypoxia and IFP were provided by DCE-MRI and related to treatment outcome.

Methods and Materials: Fifty-four cervical cancer patients treated with concurrent cisplatinbased chemoradiotherapy were studied. Low enhancing tumor volume (LETV) and peritumoral fluid flow velocity $\left(v_{0}\right)$ were used as measures of tumor hypoxia and IFP, respectively.

Results: Poor disease-free survival (DFS) and overall survival (OS) were associated with large LETV and high $v_{0}$. Multivariate analysis suggested that the prognostic power of $v_{0}$ and LETV was independent of established clinical prognostic factors and that the prognostic power of $v_{0}$ was strong compared with that of LETV. The outcome was particularly poor for patients showing high $v_{0}$ in combination with large LETV and particularly good for those having low $v_{0}$ in combination with small LETV, with 5-year DFS and OS of $13 \%$ versus $100 \%$.

Conclusions: The outcome of locally advanced cervical carcinoma seems to be influenced strongly by tumor IFP and to a lesser extent by tumor hypoxia. DCE-MRI may have the power to provide important biomarkers of the outcome of cervical cancer. 


\section{Introduction}

Locally advanced carcinoma of the uterine cervix is generally treated with cisplatin-based concurrent chemoradiotherapy, and approximately two thirds of the patients are disease-free 5 years after treatment (1-4). Poor treatment outcome is associated with a hostile physicochemical tumor microenvironment characterized by extensive hypoxia (5-7) and highly elevated interstitial fluid pressure (IFP) (8-10). It has been suggested that the outcome may be improved by personalizing the treatment, for example by giving more aggressive treatment to patients having developed a particularly hostile tumor microenvironment (11). Personalized treatment may require sensitive noninvasive methods for identifying the patients that are expected to benefit from an aggressive treatment strategy (4). Dynamic contrast-enhanced magnetic resonance imaging (DCE-MRI) could be a useful method, since it has been revealed that DCE-MRI may provide tumor biomarkers associated with poor outcome in cervical cancer (12-19).

Studies in our laboratory have revealed that a DCE-MRI parameter termed low-enhancing tumor volume (LETV) has strong prognostic power for cervical cancer patients treated with chemoradiotherapy (20). This parameter represents the tumor volume that shows low contrast enhancement during the first $60 \mathrm{~s}$ after contrast administration, and it is assumed to be a measure of the extent of tumor hypoxia. Large LETV was associated with poor disease-free and overall survival rates, and LETV was shown to provide significant and independent prognostic information for the outcome of treatment (20).

Furthermore, we have established a novel method for assessing tumor IFP from DCE-MRI series (21). In this assay, the outward peritumoral fluid flow velocity at the tumor surface $\left(v_{0}\right)$ is recorded, and the assay is based on the assumption that $v_{0}$ reflects the difference in fluid pressure between a tumor and its surrounding normal tissue and thus is an adequate measure of tumor IFP. This assumption has been validated in preclinical studies, which have shown strong correlations between $v_{0}$ measured by DCE-MRI and IFP measured by invasive met- 
hods (21). We have also revealed that $v_{0}$ is a significant prognostic factor for locally advanced cervical carcinoma (22). In that study, high $v_{0}$ was found to be associated with elevated incidence of lymph node metastases and poor disease-free and overall survival rates in patients treated with chemoradiotherapy.

Although there is significant evidence that poor outcome of cervical carcinoma is associated with severe hypoxia and interstitial hypertension in the primary tumor (5-10), no study assessing hypoxia- and IFP-related MRI parameters in the same patient cohort has been reported thus far. In the investigation reported here, LETV and $v_{0}$ were measured prior to treatment in 54 patients with locally advanced cervical cancer. The main purpose of the investigation was to provide information on the relative importance of hypoxia and IFP for the outcome of cervical carcinoma and to identify clinically useful biomarkers for guiding precision medicine in this disease. 


\section{Methods and Materials}

\section{Cervical cancer patients}

The study included 54 evaluable patients (46 squamous cell carcinomas, 7 adenocarcinomas, and 1 undifferentiated carcinoma) with untreated locally advanced cervical carcinoma (FIGO stage IB through IVA) from a cohort of 62 consecutive patients recruited to the chemoradiotherapy protocol at the Norwegian Radium Hospital during a period of 30 months. Eight patients were excluded from analysis due to poor signal-to-noise ratio and/or severe motion artifacts. The study was approved by the regional committee of medical research ethics in southern Norway and was conducted in accordance with the Declaration of Helsinki. Written informed consent was obtained from each patient.

Standard diagnostics and staging involved $T_{2}$-weighted MRI, $T_{1}$-weighted MRI, and Gd-DTPAbased DCE-MRI of the pelvis in addition to gynecological examination and biopsy. Primary tumor volume and lymph node status were determined by examining MR images in the open source dicom viewer Osirix (23). A region of interest (ROI) encompassing the tumor area was drawn in $T_{2}$-weighted images. Tumor volume was calculated in two ways: it was reconstructed and calculated from these ROls with a built-in function of Osirix and it was calculated from three orthogonal diameters using the formula of an ellipsoid. Similar results were obtained with the two methods, and the numerical values reported here refer to the latter method. Lymph node status was determined by examining the internal, external, and lower common iliac chains. A lymph node was scored as metastasis-positive when its shortest diameter in the $T_{2}$-weighted images was longer than $1.0 \mathrm{~cm}$ and the $T_{1}$-weighted images showed a contrast enhancement pattern similar to that of the primary tumor. Fine-needle aspiration cytology was used to assess lymph node status if assessment from MR images alone proved difficult.

The patients were treated with concurrent chemoradiotherapy with curative intent. External beam radiation therapy was given in 25 fractions during a period of 5 weeks to a total dose of 50 Gy to the primary tumor, parametria, and adjacent pelvic wall and 45 Gy to the rest of the pelvic region. In addition, 5 to 6 fractions of intracavitary brachytherapy with a dose of 
4.2 Gy per fraction were given to Point A. Chemotherapy with cisplatin $\left(40 \mathrm{mg} / \mathrm{m}^{2}\right)$ was given weekly with a maximum of 6 courses during the radiation therapy period.

The patients were followed up by clinical examinations every third month for the first 2 years and thereafter every sixth month. The primary endpoints were disease-free survival (DFS), defined as the time to local or distant relapse or death from any cause measured from the date of diagnosis, and overall survival (OS), defined as the interval from diagnosis to death from any cause. DFS and OS curves were generated by using the Kaplan-Meier method.

\section{Magnetic resonance imaging}

A 1.5-T whole-body scanner (Signa; General Electric) and a 4-channel phased-array surface coil were used for MRI. The entire pelvic region was scanned with an axial $T_{2}$-weighted fast spin echo sequence $\left(T R=4960 \mathrm{~ms}, \mathrm{TE}=84 \mathrm{~ms}\right.$, field of view: $20 \times 20 \mathrm{~cm}^{2}$, image matrix: $512 \times 512$, number of excitations: 1.5 , slice thickness: $5 \mathrm{~mm}$, slice spacing: $6 \mathrm{~mm}$ ). DCE-MRI was carried out by using an axial $T_{1}$-weighted spoiled gradient recalled sequence (TR $=160$ $\mathrm{ms}, \mathrm{TE}=3.5 \mathrm{~ms}, \alpha_{T 1}=90^{\circ}$, field of view: $20 \times 20 \mathrm{~cm}^{2}$, image matrix: $256 \times 256$, number of excitations: 1 , slice thickness: $5 \mathrm{~mm}$, slice spacing: $6 \mathrm{~mm}$ ). Three $T_{1}$-weighted images were acquired before a bolus of $0.1 \mathrm{mmol} / \mathrm{kg}$ Gd-DTPA was administered, and $T_{1}$-weighted images were recorded at a temporal resolution of $29 \mathrm{~s}$ after the Gd-DTPA administration. The MRI was carried out before treatment was initiated.

\section{Image processing and assessment of LETV and $v_{0}$}

Voxel-by-voxel analyses of MR images were carried out by using in-house-made software developed in Matlab. TGS (medical physicist) and KVL (radiologist) performed the analyses jointly after the patients were treated, and they were blinded to the patient outcome. Minor tumor movements during the DCE-MRI were corrected for by coordinate mapping. Relative signal intensity was calculated as the ratio of post- to pre-contrast signal intensity, using the mean of the three pre-contrast voxel values and the mean of the first and second post-contrast voxel values, corresponding to 29 and $58 \mathrm{~s}$ after contrast administration. LETV was defined as 
the volume of voxels having a post-contrast signal enhancement of $<4.0$ times the standard deviation of the pre-contrast voxel signals, and this definition is identical to that used in our previous study (20).

The procedure used to measure $v_{0}$ was similar to that reported earlier (21). Briefly, the $T_{1}$ weighted image recorded immediately after contrast administration showed a high-signalintensity rim in the tumor periphery, and this rim moved outward with time. The rim displacement $(S)$ relative to its position in the first post-contrast image was measured as a function of time $(t)$, and curves of $S(t)=S_{0}\left(1-\mathrm{e}^{-b t}\right)$ were fitted to the data by regression analysis to determine $S_{0}$ and $b$, where $S_{0}$ represents the maximum distance the interstitial fluid can flow and $b$ describes the decrease in fluid flow velocity with time. Each curve fitting was based on $~ 20$ points, one for each time frame of the DCE-MRI series. Fluid flow velocity was calculated as $v(t)=d S / d t=S_{0} b \mathrm{e}^{-b t}=v_{0} \mathrm{e}^{-b t}$, and the velocity at the tumor surface as $v_{0}=v(t=0)=S_{0} b$.

Measurements of $v_{0}$ were conducted in 1-3 central sections in each tumor, depending on the tumor size. In each section, 3-5 ROls were examined, and these ROls were positioned perpendicularly to the tumor periphery in regions where a well-defined high-signal-intensity rim could be identified throughout the entire DCE-MRI series. Mean $v_{0}$ was calculated from the $v_{0}$ of these ROls and used as a measure of tumor $v_{0}$.

\section{Statistical analysis}

The Spearman rank order correlation test was used to search for correlations between parameters. Data comparisons were performed by using the Mann-Whitney rank-sum test. Associations between treatment outcome and DCE-MRI-derived parameters were investigated by dividing the patient cohort into two groups consisting of one third and two thirds of the patients (20). Thus, the outcome of the 18 patients with the largest LETV was compared with that of the 36 patients with smallest LETV, and similar comparisons were carried out for $v_{0}$. KaplanMeier curves were compared by using the log-rank test. Univariate and multivariate Cox proportional hazard analyses were used to evaluate prognostic parameters with respect to DFS and OS. $P$ values $<0.05$ were considered significant. 


\section{Results}

Intertumor heterogeneity in LETV and $v_{0}$ and their associations with tumor volume and lymph node status

LETV and $v_{0}$ differed substantially among the primary tumors of the 54 patients included in the study. There was a significant but weak correlation between LETV and tumor volume (Fig. 1A, $P<.0001, R^{2}=0.28$ ) and between $v_{0}$ and tumor volume (Fig. $1 \mathrm{~B}, P=.0003, R^{2}=0.23$ ). Metastatic growth in regional lymph nodes was detected in 26 of the 54 patients, whereas the other 28 patients showed no evidence of lymph node metastases. LETV did not differ between the metastasis-positive and metastasis-negative patients (Fig. 2A, $P>.05$ ), whereas the metastasis-positive patients showed significantly higher $v_{0}$ than the metastasis-negative patients (Fig. 2B, $P=.0028$ ).

\section{LETV and $v_{0}$ and their associations with outcome of treatment}

The actuarial DFS and OS at 5 years were $65 \%$ and $72 \%$, respectively. Univariate Cox regression analysis showed that the two DCE-MRI-derived parameters LETV and $v_{0}$ had strong impact on DFS and OS, while similar analysis of clinical parameters revealed that DFS and OS were influenced significantly by FIGO stage and tumor volume, but not by lymph node status, tumor histology, and patient age (Table 1). Multivariate Cox regression analysis including tumor volume, FIGO stage, lymph node status, and LETV showed that none of the clinical parameters were independent prognostic factors, whereas the independent prognostic power of LETV was of borderline significance $(P=.087$ for DFS and $P=.041$ for OS). Furthermore, multivariate analyses including the clinical parameters and $v_{0}$ or the clinical parameters, LETV, and $v_{0}$ revealed that $v_{0}$ was the only independent prognostic factor and that the prognostic power of $v_{0}$ was substantial $(P<.0001)$, regardless of whether DFS or OS was considered (Table 2).

Kaplan-Meier curves for DFS and OS based on LETV or $v_{0}$ are presented in Figure 3. Patients with large LETV did worse than those with small LETV, with a 5 -year DFS of $38 \%$ versus $83 \%$ 
$(P=.0008)$ and a 5 -year OS of $39 \%$ versus $86 \%(P<.0001)$. Furthermore, DFS and OS were worse for patients with high $v_{0}$ than for those with low $v_{0}$. The 5 -year DFS was $22 \%$ versus $89 \%(P<.0001)$ and the 5 -year OS was 33\% versus $89 \%(P<.0001)$.

LETV is plotted versus $v_{0}$ in Figure $4 \mathrm{~A}$ and $\mathrm{B}$, and there was no significant correlation between these two DCE-MRI-derived parameters $(P>.05)$. Two dashed lines are drawn in the panels, and the dots above the horizontal line refer to the 18 patients having the highest LETV values, whereas the dots to the right of the vertical line refer to the 18 patients having the highest $v_{0}$ values. The white and black dots in Figure $4 \mathrm{~A}$ refer to the patients with events and those without events, respectively, where an event is local relapse, distant relapse, or patient death. In Figure 4B, green dots refer to patients with small LETV and low $v_{0}$ (Group 1, 26 patients), black dots refer to patients with large LETV and low $v_{0}$ or small LETV and high $v_{0}$ (Group 2, 20 patients), and red dots refer to patients with large LETV and high $\mathrm{v}_{0}$ (Group 3, 8 patients). Kaplan-Meier curves for DFS and OS of these three patient groups are compared in Figure 4C and D. The 5-year survival rates were 100\% (Group 1), 44\% (Group 2), and 13\% (Group 3) for DFS and 100\% (Group 1), 55\% (Group 2), and 13\% (Group 3) for OS. The survival curves were significantly different (Group 1 versus Group 2: $P<.0001$ for DFS and $P=.0002$ for OS; Group 1 versus Group 3: $P<.0001$ for DFS and OS; Group 2 versus Group 3: $P=.027$ for DFS and $P=.0018$ for OS). 


\section{Discussion}

Fifty-four patients were included in our study, and this cohort was divided into two groups, one consisting of one third of the patients (the $1 / 3$ with largest LETV when hypoxia was considered and the $1 / 3$ with highest $v_{0}$ when IFP was considered) and the other group consisting of the remaining two thirds. We have shown previously that this way of splitting a patient cohort is optimal for locally advanced cervical cancer (20), and it is consistent with the overall observation that standard first-line treatment fails in approximately one third of the patients (1).

The investigation showed that poor outcome was associated with large LETV as well as high $v_{0}$, suggesting that severe hypoxia and high IFP in the primary tumor indicate poor prognosis in cervical carcinoma. Importantly, the prognostic power of LETV and $v_{0}$ was independent of conventional prognostic factors such as lymph node status, FIGO stage, and tumor volume. Our study also revealed that the prognosis was particularly poor when the primary tumor showed both severe hypoxia and high IFP and particularly good when it had a small hypoxic volume in combination with low IFP. Moreover, the prognostic power of IFP appeared to be stronger than that of hypoxia - a possibility that needs to be validated in an independent cohort of cervical carcinoma patients.

Clinical studies reporting measurements of the extent of hypoxia and interstitial hypertension in the same tumors are sparse. However, Fyles et al measured fraction of hypoxic tissue with the Eppendorf $\mathrm{pO}_{2}$ histograph and IFP with a wick-in-needle apparatus in cervical cancer patients prior to primary radiation therapy, and IFP was found to have strong prognostic impact for DFS whereas the prognostic impact of hypoxia was of borderline significance and was limited to patients without imaging evidence of lymph node metastases (9). The results from our study are thus in accordance with the study of Fyles et al (9). Taken together, these studies provide evidence that tumor IFP may be a strong prognostic factor and the extent of tumor hypoxia a weaker prognostic factor for the outcome of cervical carcinoma. 
There are numerous biological observations providing possible explanations of a link between poor outcome of cancer and a highly abnormal physicochemical tumor microenvironment $(24,25)$. The dose of ionizing radiation required for inactivation of tumor cells is $\sim 3$-fold higher under hypoxic than under normoxic conditions, and tumor hypoxia may promote metastatic dissemination and growth by activating hypoxia-inducible factor- 1 , nuclear factor-kB, activator protein-1, and other transcription factors (26-28). High IFP may inhibit the uptake of chemotherapeutic agents in tumors (29) and cause decreased radiocurability by hypoxia-independent mechanisms (30). Moreover, preclinical studies have revealed that high IFP is associated with high metastatic propensity in cervical carcinomas (31) and other cancer types (32), possibly because the IFP-driven peritumoral interstitial fluid flow transports proteolytic enzymes, angiogenic factors, and other metastasis-promoting molecules from the primary tumor into the surrounding normal tissue $(33,34)$.

Improved outcome of locally advanced cervical carcinoma may require novel biomarkers as well as novel treatment strategies. Our study suggests that DCE-MRI has the potential to provide adequate biomarkers, as LETV may be an important biomarker for tumor hypoxia and $v_{0}$ may be an important biomarker for tumor IFP. Both biomarkers are needed because different treatment strategies may be required to overcome the problems associated with these microenvironmental abnormalities.

Potentially useful novel strategies for the treatment of advanced cervical cancer are under investigation, and some strategies focus on the use of hypoxia-targeting therapy in combination with chemoradiotherapy. Our investigation suggests that treatments targeting the causes of high IFP may be even more beneficial than hypoxia-targeting treatments, implying that significant efforts should be made to identify interstitial hypertension-targeting treatments. High IFP in tumors is primarily a consequence of high resistance to blood flow, low resistance to transvascular fluid flow, and highly impaired lymphatic drainage (35), and it has therefore been suggested that antiangiogenic treatment may be a useful modality for decreasing the IFP of tumors (29). Interestingly, studies of the effects of antiangiogenic agents in patients with advanced cervical carcinoma have been initiated, and a recent review concludes that 
antiangiogenic treatments provide promising clinical results and may have an exciting potential in the future management of cervical cancer (36).

It should be noticed, however, that our study has some significant limitations. First, the DCE$\mathrm{MRI}$ was conducted at a low temporal resolution of $29 \mathrm{~s}$. Correct assessment of $v_{0}$ requires high signal intensity, and therefore, a $T_{1}$-weighted sequence providing high signal intensity rather than high temporal resolution was chosen for this study. Because of the low temporal resolution, the peak signal intensity may have been missed, providing an uncertainty in the assessment of LETV. To reduce the magnitude of this uncertainty, LETV was calculated from the mean voxel signal intensities in the first and second post-contrast images.

Second, the calculation of LETV requires threshold values for signal intensity, and signal intensities vary depending upon the MR scanner, the strength of the magnetic field, and the MR protocol being used as well as several experimental factors, including the dose, relaxivity, and administration rate of the contrast agent. Threshold values for tumor signal enhancement can therefore not be transferred between radiology departments, implying that different imaging centers have to establish and use their own individual signal intensity threshold values.

Third, the calculation of $v_{0}$ was conducted in regions of interest positioned manually in image areas showing a well-defined high-signal-intensity rim throughout the DCE-MRI series. This procedure is not suitable for routine assessment of $v_{0}$, which may require a fully automated procedure considering the entire three-dimensional tumor surface.

In summary, this study confirmed that LETV and $v_{0}$ are significant prognostic factors for the outcome of cervical carcinoma treated with concurrent chemoradiotherapy, and moreover, multivariate analysis suggested that $v_{0}$ may be a stronger prognostic factor than LETV. DCE-MRI may have the power to provide important biomarkers of the outcome of cervical cancer patients, and consequently, DCE-MRI may be a useful imaging modality in attempts to develop novel microenvironment-targeting treatment strategies and for guiding precision medicine in cervical carcinoma. 


\section{References}

1. Green JA, Kirwan JM, Tierney JF, et al. Survival and recurrence after concomitant chemotherapy and radiotherapy for cancer of the uterine cervix: a systematic review and metaanalysis. Lancet 2001;358:781-786.

2. Barbera L, Thomas G. Management of early and locally advanced cervical cancer. Semin Oncol 2009;36:155-169.

3. Klopp AH, Eifel PJ. Biological predictors of cervical cancer response to radiation therapy. Semin Radiat Oncol 2012;22:143-150.

4. Barwick TD, Taylor A, Rockall A. Functional imaging to predict tumor response in locally advanced cervical cancer. Curr Oncol Rep 2013;15:549-558.

5. Höckel M, Schlenger K, Aral B, et al. Association between tumor hypoxia and malignant progression in advanced cancer of the uterine cervix. Cancer Res 1996;56:4509-4515.

6. Fyles AW, Milosevic M, Pintilie M, et al. Cervix cancer oxygenation measured following external radiation therapy. Int J Radiat Oncol Biol Phys 1998;42:751-753.

7. Sundfør K, Lyng H, Tropé CG, et al. Treatment outcome in advanced squamous cell carcinoma of the uterine cervix: relationships to pretreatment tumor oxygenation and vascularization. Radiother Oncol 2000;54:101-107.

8. Milosevic M, Fyles A, Hedley D, et al. Interstitial fluid pressure predicts survival in patients with cervix cancer independent of clinical prognostic factors and tumor oxygen measurements. Cancer Res 2001;61:6400-6405.

9. Fyles A, Milosevic M, Pintilie M, et al. Long-term performance of interstitial fluid pressure and hypoxia as prognostic factors in cervix cancer. Radiother Oncol 2006;80:132-137. 
10. Yeo SG, Kim JS, Cho MJ, et al. Interstitial fluid pressure as a prognostic factor in cervical cancer following radiation therapy. Clin Cancer Res 2009;15:6201-6207.

11. Vaupel P, Mayer A. Hypoxia in cancer: significance and impact on clinical outcome. Cancer Metastasis Rev 2007;26:225-239.

12. Loncaster JA, Carrington BM, Sykes JR, et al. Prediction of radiotherapy outcome using dynamic contrast enhanced MRI of carcinoma of the cervix. Int J Radiat Oncol Biol Phys 2002;54:759-767.

13. Yuh WTC, Mayr NA, Jarjoura D, et al. Predicting control of primary tumor and survival by DCE MRI during early therapy in cervical cancer. Invest Radiol 2009;44:343-350.

14. Zahra MA, Tan LT, Priest AN, et al. Semiquantitative and quantitative dynamic contrastenhanced magnetic resonance imaging measurements predict radiation response in cervix cancer. Int J Radiat Oncol Biol Phys 2009;74:766-773.

15. Mayr NA, Yuh WT, Jajoura D, et al. Ultra-early predictive assay for treatment failure using functional magnetic resonance imaging and clinical prognostic parameters in cervical cancer. Cancer 2010;116:903-912.

16. Mayr NA, Huang Z, Wang JZ, et al. Characterizing tumor heterogeneity with functional imaging and quantifying high-risk tumor volume for early prediction of treatment outcome: cervical cancer as a model. Int J Radiat Oncol Biol Phys 2012;83:972-979.

17. Andersen EKF, Hole KH, Lund KV, et al. Pharmacokinetic parameters derived from dynamic contrast enhanced MRI of cervical cancers predict chemoradiotherapy outcome. Radiother Oncol 2013;107:117-122. 
18. Torheim T, Groendahl AR, Andersen EKF, et al. Cluster analysis of dynamic contrast enhanced MRI reveals tumor subregions related to locoregional relapse for cervical cancer patients. Acta Oncol 2016;55:1294-1298.

19. Dickie BR, Rose CJ, Kershaw LE, et al. The prognostic value of dynamic contrastenhanced MRI contrast agent transfer constant $K^{\text {trans }}$ in cervical cancer is explained by plasma flow rather than vessel permeability. Br J Cancer 2017;116:1436-1443.

20. Lund KV, Simonsen TG, Hompland T, et al. Short-term pretreatment DCE-MRI in prediction of outcome in locally advanced cervical cancer. Radiother Oncol 2015;115:379-385.

21. Hompland T, Ellingsen $\mathrm{C}$, Øvrebø $\mathrm{KM}$, et al. Interstitial fluid pressure and associated lymph node metastasis revealed in tumors by dynamic contrast-enhanced MRI. Cancer Res 2012;72:4899-4908.

22. Hompland T, Lund KV, Ellingsen $\mathrm{C}$, et al. Peritumoral interstitial fluid flow velocity predicts survival in cervical cancer. Radiother Oncol 2014;113:132-138.

23. Rosset A, Spadola L, Ratib O. Osirix: an open-source software for navigating in multidimensional DICOM images. J Digit Imaging 2004;17:205-216.

24. Cairns R, Papandreou I, Denko N. Overcoming physiologic barriers to cancer treatment by molecularly targeting the tumor microenvironment. Mol Cancer Res 2006;4:61-70.

25. Horsman MR, Vaupel P. Pathophysiological basis for the formation of the tumor microenvironment. Front Oncol 2016;6:66.

26. Vaupel P. Hypoxia and aggressive tumor phenotype: implications for therapy and prognosis. Oncologist 2008;13(Suppl 3):21-26. 
27. Hill RP, Bristow RG, Fyles A, et al. Hypoxia and predicting radiation response. Semin Radiat Oncol 2015;25:260-272.

28. Schito L, Semenza GL. Hypoxia-inducible factors: master regulators of cancer progresssion. Trends Cancer 2016;2:758-770.

29. Jain RK. Delivery of molecular medicine to solid tumors. Science 1996;271:1079-1080.

30. Rofstad EK, Ruud EBM, Mathiesen B, et al. Associations between radiocurability and interstitial fluid pressure in human tumor xenografts without hypoxic tissue. Clin Cancer Res 2010;16:936-945.

31. Ellingsen $C$, Hompland T, Mathiesen B, et al. Microenvironment-associated lymph node metastasis of human cervical carcinoma xenografts. Acta Oncol 2012;51:465-472.

32. Rofstad EK, Tunheim SH, Mathiesen B, et al. Pulmonary and lymph node metastasis is associated with primary tumor interstitial fluid pressure in human melanoma xenografts. Cancer Res 2002;62:661-664.

33. Shields JD, Fleury ME, Yong C, et al. Autologous chemotaxis as a mechanism of tumor cell homing to lymphatics via interstitial flow and autocrine CCR7 signaling. Cancer Cell 2007;11:526-538.

34. Mumprecht V, Detmar M. Lymphangiogenesis and cancer metastasis. J Cell Mol Med 2009;13:1405-1416.

35. Heldin $\mathrm{CH}$, Rubin K, Pietras K, et al. High interstitial fluid pressure-an obstacle in cancer therapy. Nat Rev Cancer 2004;4:806-813.

36. Alldredge JK, Tewari KS. Clinical trials of antiangiogenesis therapy in recurrent/persistent and metastatic cervical cancer. Oncologist 2016;21:576-585. 


\section{Figure Legends}

Fig. 1. LETV, $v_{0}$, and tumor volume. LETV (A) and $v_{0}(\mathrm{~B})$ versus tumor volume for patients with locally advanced cervical cancer. Dots represent individual patients. Curves were fitted to data by linear regression analysis.

Fig. 2. LETV, $v_{0}$, and lymph node status. LETV $(\mathrm{A})$ and $v_{0}(\mathrm{~B})$ versus lymph node status for patients with locally advanced cervical cancer. Dots represent individual patients. Horizontal lines show median values. $P$ values were determined by the Mann-Whitney rank-sum test.

Fig. 3. Disease-free and overall survival. Kaplan-Meier curves for disease-free and overall survival for patients with locally advanced cervical cancer stratified by LETV (A and B) or $v_{0}$ (C and D). $P$ values were determined by the log-rank test.

Fig. 4. LETV, $v_{0}$, and treatment outcome. Fifty-four patients with locally advanced cervical cancer were treated with concurrent cisplatin-based chemoradiotherapy. (A and B) LETV versus $v_{0}$. The dots above the horizontal dashed line refer to the 18 patients having the highest LETV values, whereas the dots to the right of the vertical dashed line refer to the 18 patients having the highest $v_{0}$ values. The white and black dots in $(\mathrm{A})$ refer to the patients with events (relapse or death) and those without events, respectively. In (B), green dots refer to patients with small LETV and low $v_{0}$ (Group 1, 26 patients), black dots refer to patients with large LETV and low $v_{0}$ or small LETV and high $v_{0}$ (Group 2, 20 patients), and red dots refer to patients with large LETV and high $v_{0}$ (Group 3, 8 patients). (C and D) Kaplan-Meier curves for disease-free and overall survival for these three patient groups. 
Table 1 Univariate Cox regression analysis of clinical and DCE-MRI parameters

\begin{tabular}{lcc}
\hline & $\begin{array}{c}\text { Disease-free survival } \\
P \text { value }\end{array}$ & $\begin{array}{c}\text { Overall survival } \\
P \text { value }\end{array}$ \\
\hline Tumor volume & .032 & .0044 \\
FIGO stage & .014 & .0075 \\
Lymph node status & .096 & .17 \\
Tumor histology & .25 & .20 \\
Patient age & .36 & .20 \\
LETV & .0036 & .0006 \\
$v_{0}$ & $<.0001$ & $<.0001$ \\
\hline
\end{tabular}

Abbreviations: LETV = low enhancing tumor volume; $v_{0}=$ peritumoral fluid flow velocity.

Table 2 Multivariate Cox regression analysis of clinical and DCE-MRI parameters

\begin{tabular}{|c|c|c|c|c|c|c|}
\hline & \multicolumn{3}{|c|}{ Disease-free survival } & \multicolumn{3}{|c|}{ Overall survival } \\
\hline & \multicolumn{3}{|c|}{$P$ values } & \multicolumn{3}{|c|}{$P$ values } \\
\hline Tumor volume & $.64^{*}$ & $.053^{\dagger}$ & $.066^{\ddagger}$ & $.75^{\star}$ & $.88^{\dagger}$ & $.75^{\ddagger}$ \\
\hline FIGO stage & .12 & .29 & .26 & .14 & .35 & .33 \\
\hline Lymph node status & .34 & .50 & .48 & .78 & .71 & .72 \\
\hline LETV & .087 & & .56 & .041 & & .59 \\
\hline$v_{0}$ & & $<.0001$ & $<.0001$ & & $<.0001$ & $<.0001$ \\
\hline
\end{tabular}

Abbreviations: LETV = low enhancing tumor volume; $v_{0}=$ peritumoral fluid flow velocity. Based on *clinical parameters (tumor volume, FIGO stage, lymph node status) and LETV, ${ }^{\dagger}$ clinical parameters and $v_{0}$, and ${ }^{ \pm}$clinical parameters, LETV, and $v_{0}$. 
A

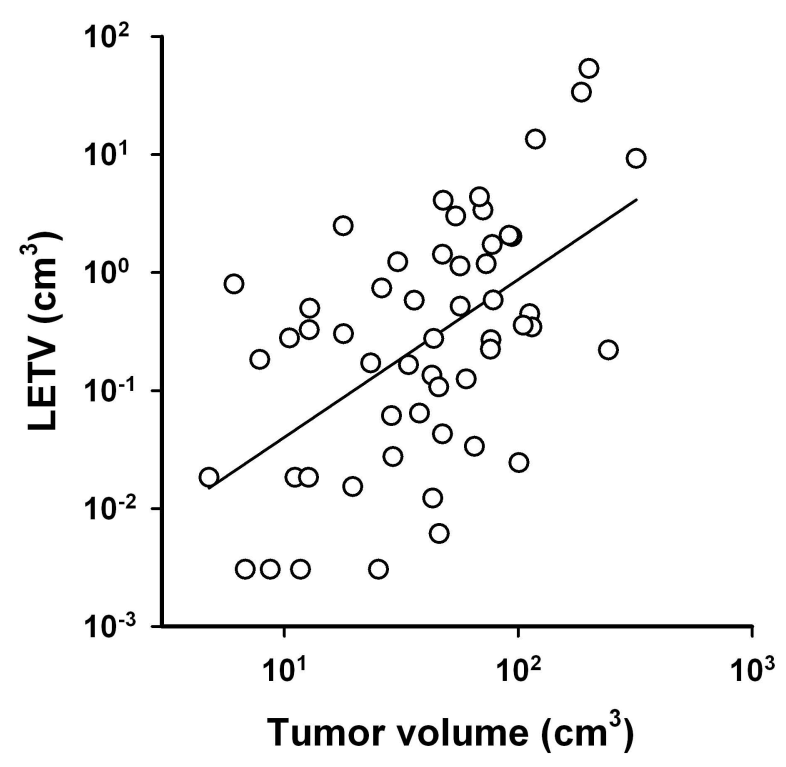

B

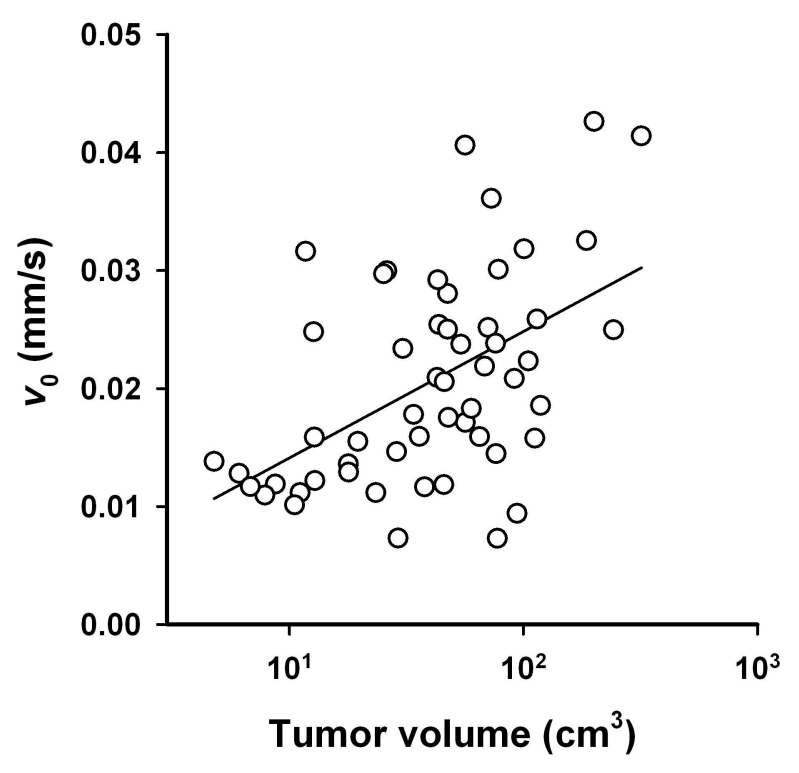

Figure 1 
A

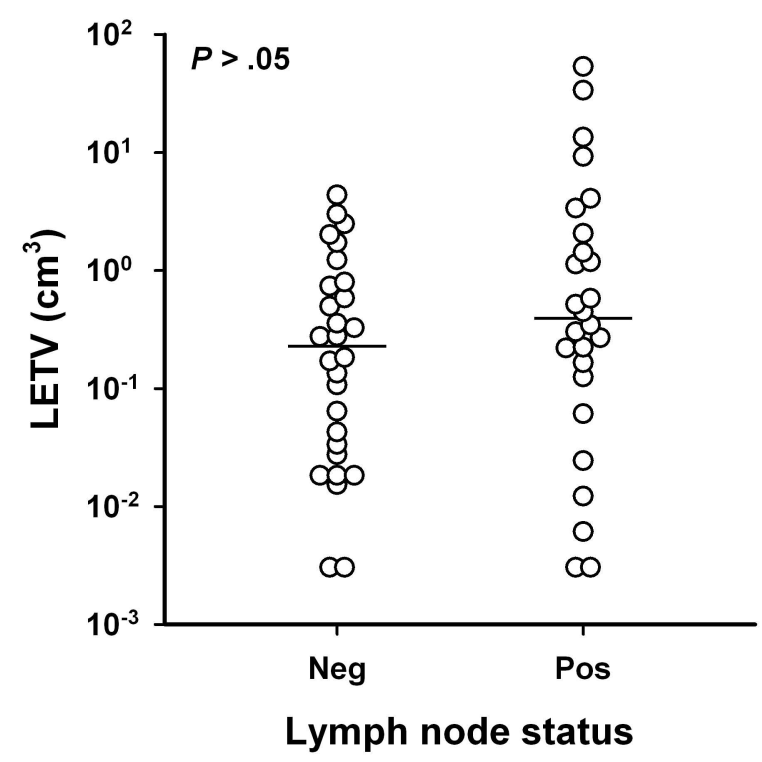

B

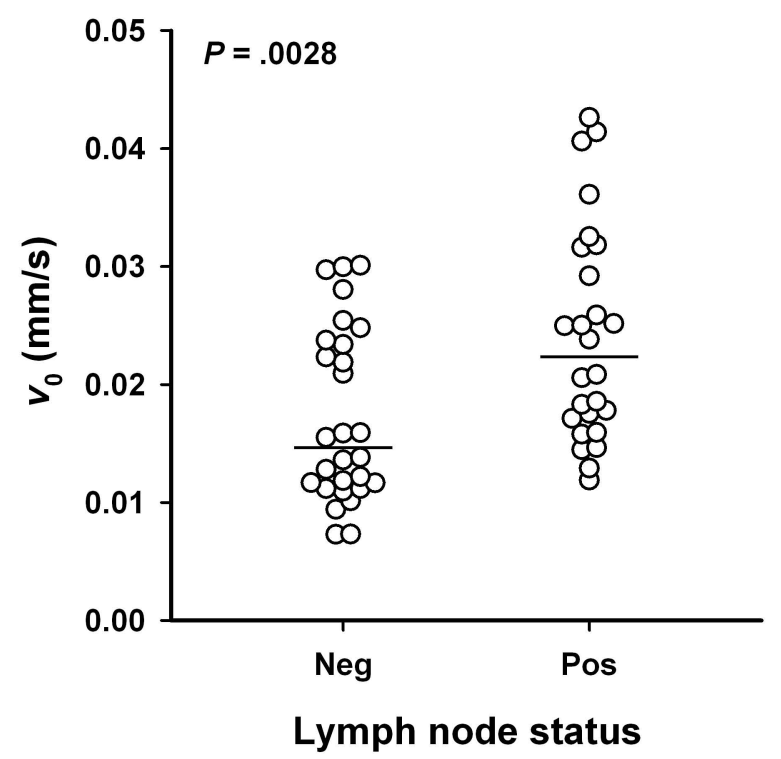

Figure 2 
A

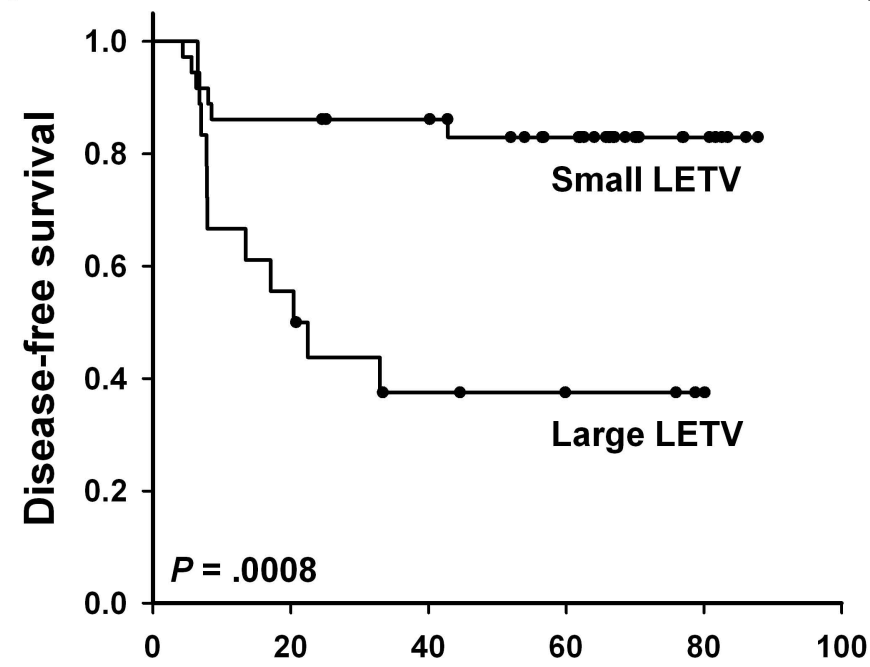

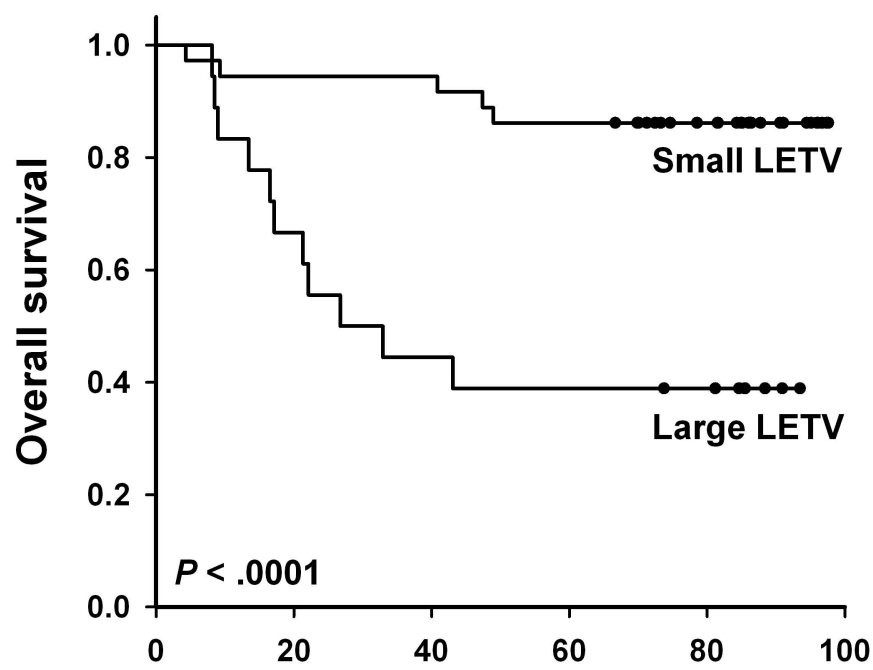

Time to death (months)

Time to recurrence or death (months)

Number of patients at risk

Small LETV $36 \quad 31$

Large LETV 18

C

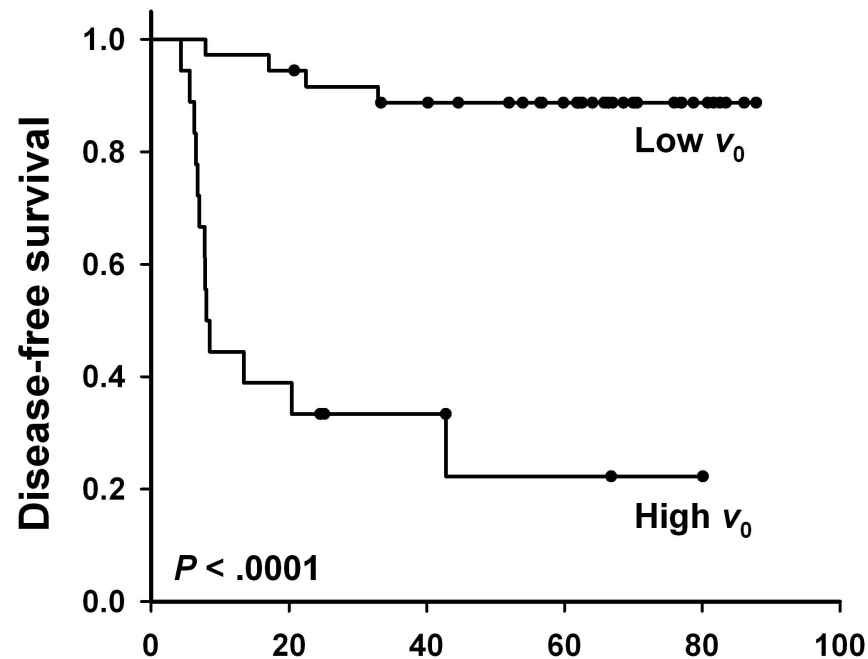

Time to recurrence or death (months)
Number of patients at risk

$\begin{array}{rrrrr}\text { Small LETV } 36 & 34 & 34 & 31 & 21 \\ \text { Large LETV } 18 & 12 & 8 & 7 & 6\end{array}$

D

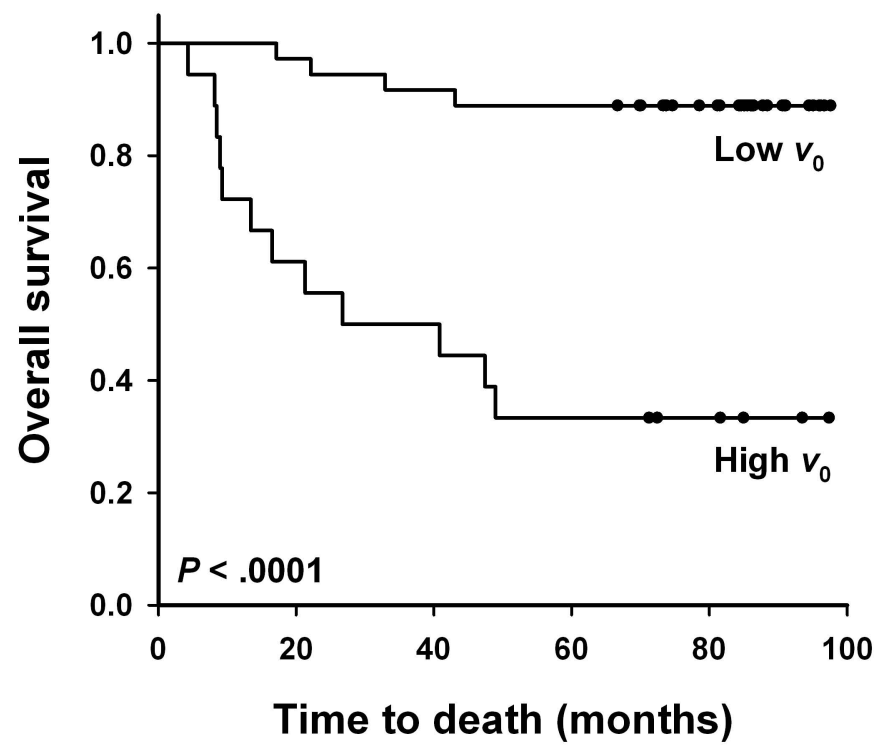

Number of patients at risk

$\begin{array}{rrrrrr}\text { Low } v_{0} & 36 & 35 & 33 & 32 & 23 \\ \text { High } v_{0} & 18 & 11 & 9 & 6 & 4\end{array}$

Figure 3 
A

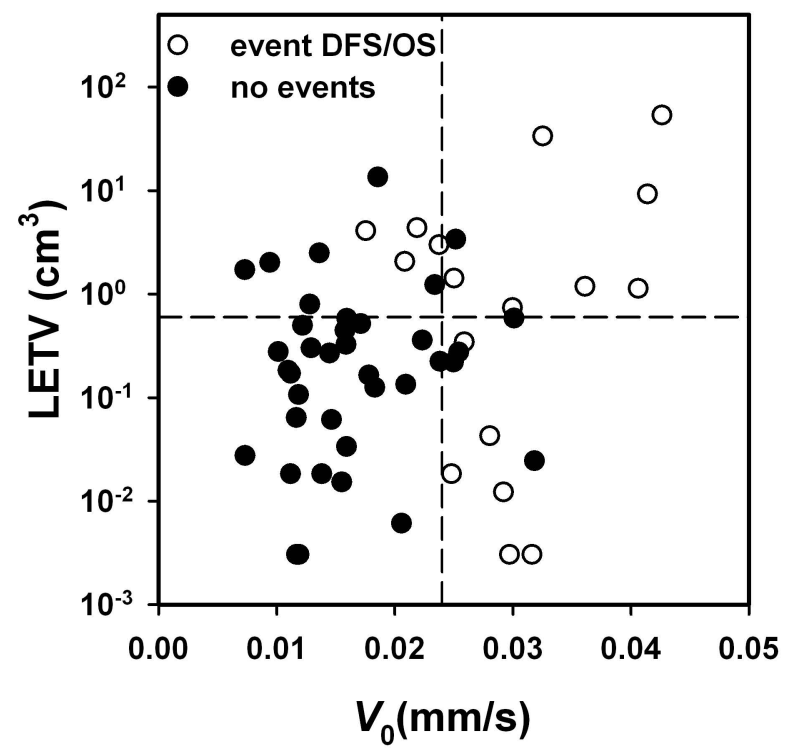

C

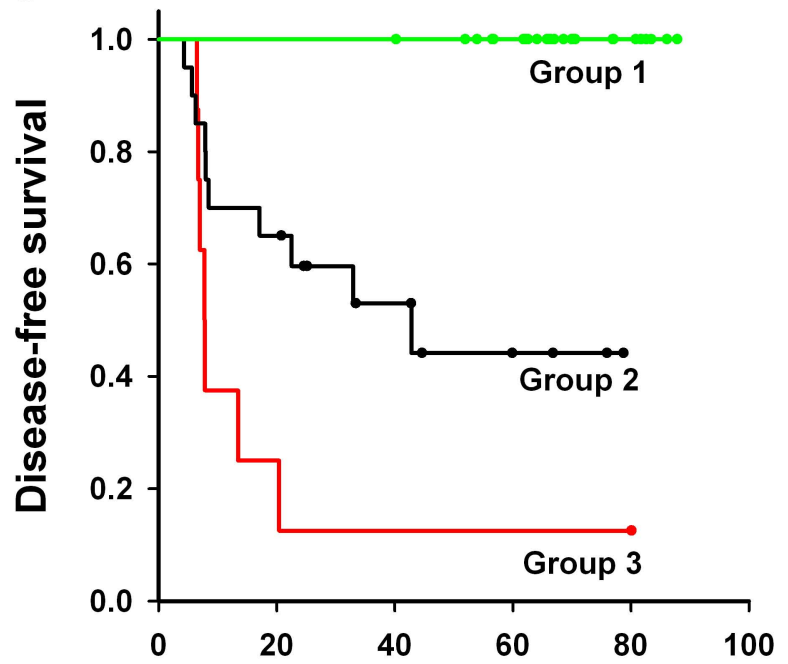

B

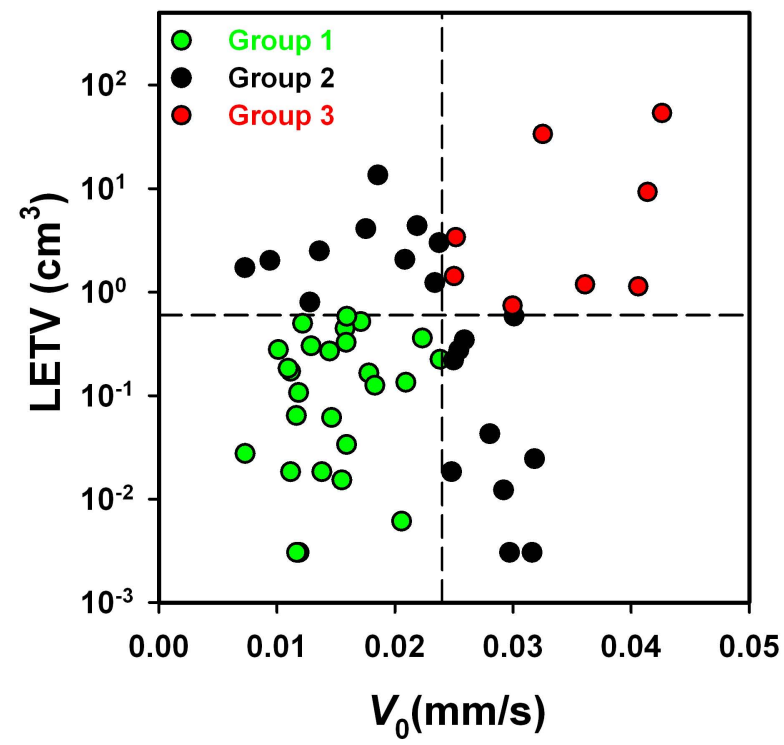

D

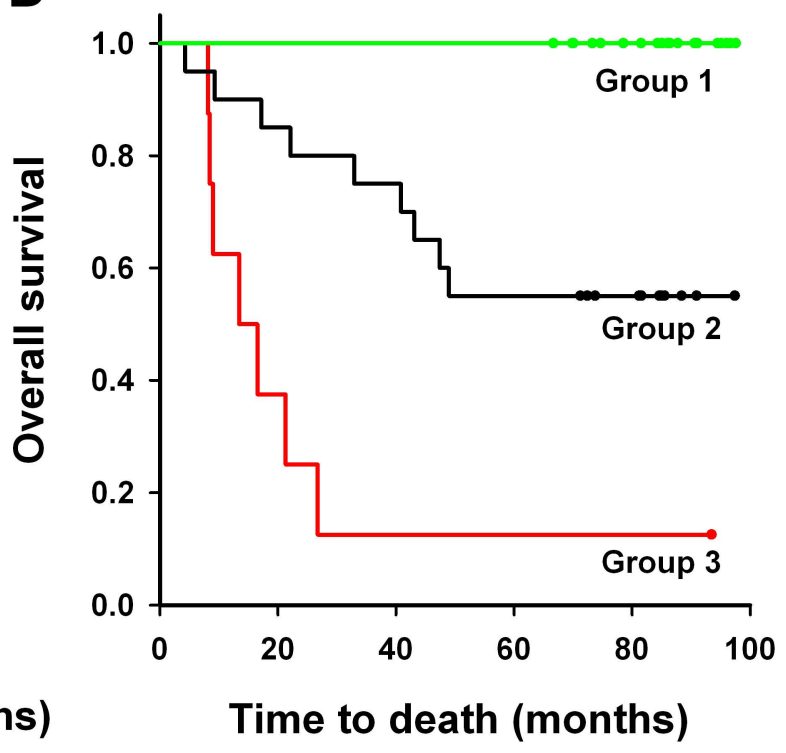

Number of patients at risk

$\begin{array}{cccccc}\text { Group 1 } & 26 & 26 & 26 & 21 & 6 \\ \text { Group 2 } & 20 & 13 & 7 & 3 & 0 \\ \text { Group 3 } & 8 & 2 & 2 & 2 & 1\end{array}$

Number of patients at risk

$\begin{array}{cccccc}\text { Group 1 } & 26 & 26 & 26 & 26 & 18 \\ \text { Group 2 } & 20 & 17 & 15 & 11 & 8 \\ \text { Group 3 } & 8 & 3 & 1 & 1 & 1\end{array}$

Figure 4 